\title{
A Note on the Normalizer Property for Integral Group Rings of Finite Groups
}

\author{
Z. Hua, J.K. Hai \\ College of Mathematics, Qingdao University \\ Qingdao, China
}

\begin{abstract}
Let $G$ be a finite group with an abelian Sylow 2 subgroup. Let $N$ be a nilpotent normal subgroup of index in $G$ at most 5 . Then the normalizer property holds for $G$. The result generalizes a well-known result due to $\mathrm{Li}$, Sehgal and Parmenter.
\end{abstract}

Keywords-coleman automorphisms; class-preserving automorphisms; normalizer property

\section{INTRODUCTION}

Let $G$ be a finite group and $Z G$ be the integral group ring of $G$ over $Z$. We denote by $Z(U(Z G))$ the center of $U(Z G)$, where $U(Z G)$ is the group of units in $Z G$. It is an classical subject to investigate the normalizer $N_{U(Z G)}(G)$ of $G$ in $U(Z G)$.Obviously, $N_{U(Z G)}(G) \geq G Z(U(Z G))$. A question (Problem 43 in Sehgal[12]) naturally arising is whether $N_{U(Z G)}(G)=G Z(U(Z G))$ when $G$ is finite. If this equality holds, we say that the normalizer property holds for $G$. Historically, the first positive result for this question was due to Coleman[1], who proved that the normalizer property holds for all nilpotent groups of finite order. Later, Jackowski, Marciniak[7] generalized this result to finite groups with a normal Sylow 2 -subgroup. In particular, the normalizer property holds for finite group of odd order. It was Mazur who first noticed that there are close connections between the normalizer problem and the isomorphism problem(for details, see [9-11]). Based on Mazur's work, among other things, Hertweck[3] presented a first counterexample to the normalizer problem, then a first counterexample to the isomorphism problem. Nevertheless, it is still of interest to investigate for which groups the normalizer property holds.

In order to investigate the normalizer problem, as in [5], we introduce some kinds of automorphisms of finite group $G$.

$A u t_{c}(G)$ consists of those $\sigma \in \operatorname{Aut}(G)$ which maps every element of $G$ to its conjugate (class-preserving automorphisms' for short);

$A u t_{C o l}(G)$ consists of those $\sigma \in \operatorname{Aut}(G)$ such that for any Sylow subgroup $P$ of $G$ ('Coleman automorphisms' for short); $A u t_{Z}(G)$ consists of those $\sigma \in A u t(G)$ which induces an inner automorphism of the integral group ring $Z G$.

We use $\operatorname{Inn}(G)$ to denote the inner automorphism group of $G$. It is easy to see that $\operatorname{Inn}(G) \leq A u t_{c}(G)$, $\operatorname{Inn}(G) \leq A u t_{C o l}(G)$ and $\operatorname{Inn}(G) \leq A u t_{Z}(G)$. We set $\operatorname{Out}_{Z}(G) \quad=\quad \operatorname{Aut}_{c}(G) / \operatorname{Inn}(G)$, $\mathrm{Out}_{\mathrm{Col}}(G) \quad=\quad A u t_{C o l}(G) / \operatorname{Inn}(G) \quad$ and $\operatorname{Out}_{Z}(G)=\operatorname{Aut}_{Z}(G) / \operatorname{Inn}(G)$. It is well-known that $N_{U(Z G)}(G)=G Z(U(Z G))$ is equivalent to $\operatorname{Out}_{Z}(G)=1$. Moreover, we have $\mathrm{Out}_{Z}(G) \leq \mathrm{Out}_{c}(G) \bigcap \mathrm{Out}_{\mathrm{Col}}(G)$. In addition, Krempa showed that $\operatorname{Out}_{Z}(G)$ is an elementary abelian 2 -group(a proof can be found in [7,Theorem 3.2]). Thus, if one can show that $\mathrm{Out}_{c}(G) \cap O \mathrm{Out}_{\mathrm{Col}}(G)$ is of odd order, then $\operatorname{Out}_{Z}(G)=1$, the normalizer property holds for such group $G$.

Recently, lots of work related to the normalizer property had been done. For instance, Li, Sehgal and Parmenter $[8$, Theorem 2] have proved that if a finite group $G$ has an abelian subgroup of index 2 , then the normalizer property holds for $G$.

Motivated by this result, in this article, we will show that if $\$ \mathrm{G} \$$ has a nilpotent subgroup of index 2 , then the normalizer property holds for $G$. Our main result is the following:

Main Theorem Let $G$ be a finite group with an abelian Sylow 2 -subgroup. Let $N$ be a nilpotent normal subgroup of index in $G$ at most 5 . Then the normalizer property holds for $G$.

Throughout this paper, all groups under consideration are finite. Let $N$ be a (normal) subgroup of $G$, and $\sigma$ an automorphism of $G$. If induces an automorphism of $N$ or $G / N$, which will be denoted by $\left.\sigma\right|_{N}$ or $\left.\sigma\right|_{G / N}$,respectively. For a fixed element $x \in G, \operatorname{conj}(x)$ is 
used to denoted the inner automorphism induced by $x$, i.e., $\operatorname{conj}(x)(g)=g^{x}$ for all $g \in G$.

\section{PRELIMINARY RESULTS}

Lemma 2.1([6], Lemma 2). Let $\varphi \in \operatorname{Aut}(G)$ be of $p$ power order with $p$ a prime. Suppose that there is a normal subgroup $N$ of $G$ such that $\varphi$ fixes $N$ elementwise, and that $\varphi$ induces the identity on the quotient group $G / N$. Then $\varphi$ induces the identity on $G / O_{P}(Z(N))$. Further, if $\varphi$ fixes elementwise a Sylow $p$-subgroup of $G$, then $\varphi$ is an inner automorphism of $G$.

Lemma 2.2([12], Theorem 2.7). Let $G$ be a finite group. Then $Z G$ has only trivial units if and only if $G$ is an abelian group of exponent $2,3,4,6$ or $G \cong E \times Q_{8}$, where $Q_{8}$ is the quaternion group of order 8 and $E$ is an elementary abelian 2 -group.

Lemma 2.3([4], Theorem 1.1). Let $N$ be normal subgroup of $G$ such that $Z(G / N)$ has only trivial units. Then for any $\sigma \in A u t_{Z}(G)$, there is $\gamma \in \operatorname{Inn}(G)$ such that $o \gamma$ induces a Coleman automorphism of $N$.

Lemma 2.4([2], Corollary 3). Let $N \triangleleft G$ and let $p$ be a prime which does not divide the order of $G / N$. Then the following hold.

(1) If $\sigma \in \operatorname{Aut}(G)$ is a class-preserving or a Coleman automorphism of $G$ of $p$-power order, then $\sigma$ induces a class-preserving or a Coleman automorphism of $N$, respectively;

(2) If $O u t_{c}(N)$ or $O u t_{C o l}(N)$ is a $p^{\prime}$-group, then so is $\mathrm{Out}_{c}(G)$ or $\mathrm{Out}_{\mathrm{Col}}(G)$.

Lemma 2.5([7]). Suppose that a finite group $G$ has a normal Sylow 2 -subgroup. Then the normalizer property holds for $G$.

Lemma 2.6([7]). Let $\mathrm{S}$ be a fixed Sylow 2 -subgroup of a finite group $G$ and $I_{S}$

$\left\{\varphi_{u}\left|u \in N_{U(Z G)}(G), \varphi_{u}\right|_{S}=i d, \varphi_{u}{ }^{2}=i d\right\} \quad$

Suppose that $I_{S} \subseteq \operatorname{Inn}(G)$. Then the normalizer property holds for $G$.

Lemma 2.7([1]). Suppose that $p$ is a $p$-subgroup of a finite group $G$ and that $u \in N_{U(Z G)}(G)$. Then there exists $y \in G$ such that $\varphi_{u}(g)=u^{-1} g u=y^{-1} g y$ for all $g \in P$.
Lemma 2.8([13, lemma 1$])$. Let $G$ be a finite group and $u \in N_{U(Z G)}(G)$,

Then $\varphi_{u}(g)$ and $g$ are conjugate in $G$.

Lemma 2.9([14, Theorem 3.1]). Let $G$ be a finite group with abelian Sylow 2 -subgroups. Suppose that there exists a nilpotent normal subgroup $N$ of $G$, such that a Sylow 2 -subgroups of $G / N$ is normal. Then $A u t_{c}(G)=$

$\operatorname{Inn}(G)$. In particular, the normalizer property holds for $G$.

Lemma 2.10([5]). If the Sylow 2 -subgroups of $G$ are dihedral or general$G$.

ized quaternion, then the normalizer property holds for

Lemma 2.11. Let $N$ be a normal subgroup of $G$, and $\sigma \in \operatorname{Aut}(G)$ be a non-inner automorphism of $p$-power order with $p$ a prime. If $\sigma$ induces an inner automorphism of $G / N$, then there exists $\gamma \in \operatorname{Inn}(G)$ such that

$$
\left.\gamma \sigma\right|_{G / N}=\left.i d\right|_{G / N}
$$

and $\gamma \sigma$ is still a non-inner automorphism of $p$-power order.

Proof: $\quad$ For convenience, we write $\bar{G}=G / N$ and $\bar{g}=g N$ for any $g \in G$.

Since $\sigma$ induces an inner automorphism of $G / N$, we assume that

$$
\left.\sigma\right|_{\bar{G}}=\operatorname{conj}(\bar{x})=\left.\operatorname{conj}(x)\right|_{\bar{G}}
$$

Let $O(\sigma)=\mathrm{p}^{i}$, where $i$ is a positive inter. Let $\beta=\operatorname{conj}(x)$, then ,

$$
\left.\beta^{-1} \sigma\right|_{\bar{G}}=\left.i d\right|_{\bar{G}}
$$

Let $\left(\beta^{-1} \sigma\right)^{n}$ be the $p$-part of $\beta^{-1} \sigma$, where $n$ is positive integer with $(n, p)=1$. It follows that there exist $s, t \in Z$ such that $s n+t p^{i}=1$. Then it is obvious that $\left(\beta^{-1} \sigma\right)^{s n}$ is of $p$-power order and $\left.\left(\beta^{-1} \sigma\right)^{s n}\right|_{\bar{G}}=\left.i d\right|_{\bar{G}}$. Since $\operatorname{Inn}(G) \unlhd A u t(G)$, it follows that there exists an inner automorphism

$\gamma \in \operatorname{Inn}(G)$, such that $\left(\beta^{-1} \sigma\right)^{s n}=\gamma \sigma^{s n}=\gamma \sigma^{1-t p^{i}}=\gamma \sigma$ and thus

$$
\left.\gamma \sigma\right|_{\bar{G}}=\left.\left(\beta^{-1} \sigma\right)^{s n}\right|_{\bar{G}}=\left.i d\right|_{\bar{G}}
$$


Lemma 2.12. Let $N$ be a subgroup of $G$, and $\sigma \in \operatorname{Aut}(G)$ be of $p$-power order with $p$ a prime. If $\sigma$ fixed $N$, and $\left.\sigma\right|_{N}=\left.\operatorname{conj}(x)\right|_{N}$ for some $x \in G$,then there a $p$-element $y \in G$, such that $\left.\sigma\right|_{N}=\left.\operatorname{conj}(y)\right|_{N}$.

Proof Let $O(\sigma)=\mathrm{p}^{i}, O(\mathrm{x})=\mathrm{p}^{j} t$, where $i, j, t \in \mathbb{N}$ and $(p, t)=1$.

Set $k=\max \{i, j\}$. Since $\left(\mathrm{p}^{k}, \mathrm{t}\right)=1$, it follows that there exists $u, v \in \mathbb{Z}$,

Such that $u p^{k}+v t=1$. Write $y=x^{v t}$. Then it is obvious that $y$ is a $p$-element. For any $z \in N$, since $z=z^{\sigma^{u p^{k}}}=z^{x^{u p^{k}}}$, it follows that

$$
\begin{aligned}
& z^{\sigma}=z^{x}=z^{x^{u p^{k}+v t}}=\left(z^{z^{u p^{k}}}\right)^{x^{v t}}=z^{x^{v t}}=z^{y} \\
& \text { and thus }\left.\sigma\right|_{N}=\left.\operatorname{conj}(y)\right|_{N} .
\end{aligned}
$$

\section{PROOF OF MAIN THEOREM}

Proposition 3.1. Let $G$ be a finite group with an abelian Sylow 2 -subgroup. Let $N$ be a normal subgroup of $G$ such that $Z(G / N)$ has only trivial units. If $\mathrm{Out}_{\mathrm{Col}}(N)$ is a $2^{\prime}$-group, then the normalizer property holds for $G$.

Proof Let $\sigma \in A u t_{Z}(G)$. It suffices to show that $\sigma \in \operatorname{Inn}(G)$. By Krempa's result, we may assume that $\sigma$ is of 2 power order. By Lemma 2.3, $\sigma$ can be modified by an inner automorphism of $G$ such that $\left.\sigma\right|_{N} \in$ $A u t_{C o l}(N)$. By assumption $O u t_{C o l}(N)$ is a $2^{\prime}$-group, so we have $\left.\sigma\right|_{N} \in \operatorname{Inn}(N)$. That is, there exits $x \in N$ such that $\left.\sigma\right|_{N}=\left.\operatorname{conj}(x)\right|_{N}$. Replacing $\sigma$ by a suitable odd power of $\sigma \operatorname{conj}\left(x^{-1}\right)$, we may assume that

$$
\left.\sigma\right|_{N}=\left.i d\right|_{N}
$$

Since $Z(G / N)$ has only trivial units, it follows from a well-known result due to Higman(see [15, Theorem(2.7)]) that $G / N$ is either an abelian group of exponent $1,2,3$, 4,6 or $G \cong E \times Q_{8}$ with $E$ being an elementary abelian 2 -group and $Q_{8}$ being the quaternion group of order 8 . But the latter case can not occur under our assumption that $\mathrm{G}$ has an abelian Sylow 2 -subgroup. In fact, if $G / N \cong E \times Q_{8}$, then $G / N$ is a nonabelian 2 -group. Hence there exits a(any) Sylow 2 -subgroup of $G$, say $P$, such that $G / N=P N / N=P / N \cap P$ is abelian, a contradiction. Therefore $G / N$ is abelian. Since $\sigma$ preserves conjugacy classes of $G$, obviously it induces the identity on $G / N$,that is

$$
\left.\sigma\right|_{G / N}=\left.i d\right|_{G / N}
$$

Note that $\sigma$ acts naturally on $\operatorname{Syl}_{2}(G)$, the set of all Sylow 2 -subgroups of $G$. Since the order of $\sigma$ is a power of 2 and the number of the Sylow 2 -subgroups of $G$ is odd, it follows that there is a Sylow 2 -subgroup of $G$, say $P$, such that $P^{\sigma}=P$.Further, since the restriction of $\sigma$ to $P$ equals the restriction of some inner automorphism of $G$,there exists $y \in G$ such that $\left.\sigma\right|_{P}$ $=\left.\operatorname{conj}(y)\right|_{P}$. Without loss of generality, we may assume that $y$ is a 2 -element. Thus we have $P=P^{\sigma}=P^{y}$, i.e., $y \in N_{G}(P)$.It follows that $y \in P$ since $y$ is a 2 element. But by assumption $P$ is a abelian, so we have

$$
\left.\sigma\right|_{P}=\left.\operatorname{conj}(y)\right|_{P}=\left.i d\right|_{P}
$$

Then by (1), (2) and (3), it follows from Lemma 2.1 that $\sigma \in \operatorname{Inn}(G)$. This completes the proof.

Proof of Main Theorem If $|G / N|=1$, then $G$ is nilpotent and thus the assertion holds.

If $|G / N|=2$ or 4 , then $G / N$ is abelian group of exponent 2 or 4 . By Lemma 2.2, $Z(G / N)$ has only trivial units. Hence the assertion follows from Proposition 3.1.

$$
\text { If }|G / N|=5 \text {, then } 2 \| G / N \mid \text {. Since } N \text { is nilpotent, it }
$$
follows that $\operatorname{Out}_{C o l}(N)$ is a $2^{\prime}$-group and thus by Lemma 2.4, Out $_{C o l}(G)$ is a $2^{\prime}$-group. This implies that $\operatorname{Out}_{Z}(G)=1$, namely, the normalizer property holds for such group $G$. This completes the proof.

\section{REFERENCES}

[1] Coleman, D. B., on the modular group ring of a $p$-group. Proc. Amer. Math. Soc. 5, 511-514 (1964)

[2] Hertweck, M., Coleman automorphisms of finite groups. Math. Z. 242, 203-215 (2002)

[3] Hertweck, M., A counterexample to the isomorphism problem for integral group rings. Ann. Math. 154, 115-138 (2001)

[4] J. K. Hai and Z. X. Li, The normalizer property for integral group rings of finite groups with abelian Sylow 2-subgroups, Acta Math. Sinica, Chinese Series 55, 187-192 (2012)

[5] Hertweck, M., Class-preserving Coleman automorphisms of finite groups. Monatsh. Math. 136, 1-7 (2002)

[6] Hertweck, M., Class-preserving automorphisms of finite groups. J. Algebra 241, 1-26 (2001) 
[7] Jackowski, S., Marciniak, Z. S.: Group automorphisms inducing the identity map on cohomology. J. Pure Appl. Algebra 44, 241-250 (1987)

[8] Li, Y., Sehgal, S. K., Parmenter, M. M.: On the normalizer property for integral group rings. Comm. Algebra 27,4217-4223 (1999)

[9] Mazur, M., Automorphisms of finite groups. Comm. Algebra 22, 6259-6271 (1994)

[10] Mazur, M., on the isomorphism problem for infinite group rings. Expositiones Mathematicae 13, 433-445 (1995)

[11] Mazur, M., The normalizer of a group in the unit group of its group ring. J. Algebra 212, 175-189 (1999)

[12] Sehgal, S. K., Units in integral group rings. Longman Scientific and Technical Press, Harlow (1993)

[13] Petit Lobao, T. and Sehgal, S. K. The normalizer property for integral group rings of complete momonial groups. Comm. Algebra 31 29712983 (2003).

[14] Jurianns, S. O., de Miranda, J. M. and Roberio, J. R., Automorphisms of finite groups. Comm. Algebra 32 1705-1714 (2004) .

[15] Li, Y., Sehgal, S. K. and Paementer, M. M., On the normalizer property for integral group rings. Comm. Algebra 27 4217-4223 (1999) . 